\title{
Bicomplex Tetranacci and Tetranacci-Lucas Quaternions
}

\author{
Yüksel Soykan \\ Department of Mathematics, Art and Science Faculty, \\ Zonguldak Bülent Ecevit University, 67100, Zonguldak, Turkey
}

\begin{abstract}
In this paper, we introduce the bicomplex Tetranacci and Tetranacci-Lucas quaternions. Moreover, we present Binet's formulas, generating functions, and the summation formulas for those bicomplex quaternions.
\end{abstract}

2010 Mathematics Subject Classification. 11B39, 11B83, 17A45, 05A15.

Keywords. bicomplex Tetranacci numbers, bicomplex quaternions, bicomplex Tetranacci quaternions, bicomplex Tetranacci-Lucas quaternions.

\section{Introduction}

In this paper, we define bicomplex Tetranacci and bicomplex Tetranacci-Lucas quaternions by combining bicomplex numbers and Tetranacci, Tetranacci-Lucas numbers and give some properties of them. Before giving their definition, we present some information on bicomplex numbers and also on Tetranacci and Tetranacci-Lucas numbers.

The bicomplex numbers (quaternions) are defined by the four bases elements $1, i, j, i j$ where $i, j$ and $i j$ satisfy the following properties:

$$
i^{2}=-1, j^{2}=-1, i j=j i
$$

A bicomplex number can be expressed as follows:

$$
q=a_{0}+i a_{1}+j a_{2}+i j a_{3}=\left(a_{0}+i a_{1}\right)+j\left(a_{2}+i a_{3}\right)=z_{0}+j z_{1}
$$

where $a_{0}, a_{1}, a_{2}, a_{3}$ are real numbers and $z_{0}, z_{1}$ are complex numbers. So the set of bicomplex number is

$$
\mathbb{B C}=\left\{z_{0}+j z_{1}: z_{0}, z_{1} \in \mathbb{C}, j^{2}=-1\right\}
$$


Moreover, for any bicomplex numbers $q=a_{0}+i a_{1}+j a_{2}+i j a_{3}$ and $p=b_{0}+i b_{1}+j b_{2}+i j b_{3}$ and skaler $\lambda \in \mathbb{R}$, the addition, substraction and multiplication with scalar are defined as componentwise, i.e

$$
\begin{aligned}
q+p & =\left(a_{0}+b_{0}\right)+i\left(a_{1}+b_{1}\right)+j\left(a_{2}+b_{2}\right)+i j\left(a_{3}+b_{3}\right) \\
q-p & =\left(a_{0}-b_{0}\right)+i\left(a_{1}-b_{1}\right)+j\left(a_{2}-b_{2}\right)+i j\left(a_{3}-b_{3}\right), \\
\lambda q & =\lambda a_{0}+i \lambda a_{1}+j \lambda a_{2}+i j \lambda a_{3}
\end{aligned}
$$

respectively, and product (multiplication) is defined as follows:

$$
\begin{aligned}
q \times p= & \left(a_{0} b_{0}-a_{1} b_{1}-a_{2} b_{2}+a_{3} b_{3}\right) \\
& +i\left(a_{0} b_{1}+a_{1} b_{0}-a_{2} b_{3}-a_{3} b_{2}\right) \\
& +j\left(a_{0} b_{2}-a_{1} b_{3}+a_{2} b_{0}-a_{3} b_{1}\right) \\
& +i j\left(a_{0} b_{3}+a_{1} b_{2}+a_{2} b_{1}+a_{3} b_{0}\right) .
\end{aligned}
$$

Multiplication of basis elements of bicomplex numbers can be done according to following Table 1:

Table 1. Multiplication Table

\begin{tabular}{|l|l|l|l|l|}
\hline$\times$ & 1 & $i$ & $j$ & $i j$ \\
\hline 1 & 1 & $i$ & $j$ & $i j$ \\
\hline$i$ & $i$ & -1 & $i j$ & $-j$ \\
\hline$j$ & $j$ & $i j$ & -1 & $-i$ \\
\hline$i j$ & $i j$ & $-j$ & $-i$ & -1 \\
\hline
\end{tabular}

There are three different conjugations (involutions) for bicomplex numbers, namely

$$
\begin{aligned}
q_{i}^{*} & =a_{0}-i a_{1}+j a_{2}-i j a_{3}=\overline{z_{0}}+j \overline{z_{1}}, \\
q_{j}^{*} & =a_{0}+i a_{1}-j a_{2}-i j a_{3}=z_{0}-j z_{1}, \\
q_{i j}^{*} & =a_{0}-i a_{1}-j a_{2}+i j a_{3}=\overline{z_{0}}-j \overline{z_{1}},
\end{aligned}
$$

for $q=a_{0}+i a_{1}+j a_{2}+i j a_{3}$. The squares of norms of the bicomplex numbers which arise from the definitions of conjugations are given by

$$
\begin{aligned}
N_{i}^{2}(q) & =\left|q_{i} \times q_{i}^{*}\right|:=\left|a_{0}^{2}+a_{1}^{2}-a_{2}^{2}-a_{3}^{2}+2 j\left(a_{0} a_{2}+a_{1} a_{3}\right)\right|, \\
N_{j}^{2}(q) & =\left|q_{j} \times q_{j}^{*}\right|:=\left|a_{0}^{2}+a_{1}^{2}-a_{2}^{2}-a_{3}^{2}+2 i\left(a_{0} a_{1}+a_{2} a_{3}\right)\right|, \\
N_{i j}^{2}(q) & =\left|q_{i j} \times q_{i j}^{*}\right|:=\left|a_{0}^{2}+a_{1}^{2}+a_{2}^{2}+a_{3}^{2}+2 i j\left(a_{0} a_{3}-a_{2} a_{1}\right)\right| .
\end{aligned}
$$

We now give some basic informations on quaternions. Quaternions were formally invented by Irish mathematician W. R. Hamilton (1805-1865) as an extension to the complex numbers and for some background 
about this type of hypercomplex numbers we refer the works, for example, in $[3,6,23]$. The field $\mathbb{H}$ of quaternions is a four-dimensional non-commutative $\mathbb{R}$-field generated by four base elements $1, i, j$ and $k$ that satisfy the following rules:

$$
i^{2}=j^{2}=k^{2}=i j k=-1
$$

and

$$
i j=k=-j i, j k=i=-k j, k i=j=-i k .
$$

Briefly $\mathbb{B C}$, the set of bicomplex numbers, has the following properties:

- Quaternions and bicomplex numbers are generalizations of complex numbers, but one difference between them is that quaternions are non-commutative, whereas bicomplex numbers are commutative.

- Real quaternions are non-commutative, and don't have zero divisors and non-trivial idempotent elements. But bicomplex numbers are commutative, have zero divisors and non-trivial idempotent elements:

$$
\begin{aligned}
i j & =j i \\
(i+j)(i-j) & =i^{2}-i j+j i-j^{2}=0, \\
\left(\frac{1+i j}{2}\right)^{2} & =\frac{1+i j}{2} .
\end{aligned}
$$

- All above norms are isotropic. For example, for $N_{i}$, we calculate $N_{i}(q)$ for $q=1+i j$ as

$$
N_{i}^{2}(1+i j)=(1+i j)(1-i j)=1^{2}-i j+i j-(i j)^{2}=0 .
$$

- $\mathbb{B C}$ is a real vector space with the addition of bicomplex numbers and the multiplication of a bicomplex number by a real scalar.

- $\mathbb{B C}$ forms a commutative ring with unity which contains $\mathbb{C}$.

- $\mathbb{B C}$ forms a two-dimensional algebra over $\mathbb{C}$, and since $\mathbb{C}$ is of dimension two over $\mathbb{R}$, the bicomplex numbers are an algebra over $\mathbb{R}$ of dimension four.

- $\mathbb{B C}$ is a real associative algebra with the bicomplex number product $x$.

For more details about these type of numbers (quaternions), we refer to, for example, the works $[8,18]$, among others.

Tetranacci sequence $\left\{M_{n}\right\}_{n \geq 0}$ and Tetranacci-Lucas sequence $\left\{R_{n}\right\}_{n \geq 0}$ are defined by the fourth-order recurrence relations

$$
M_{n}=M_{n-1}+M_{n-2}+M_{n-3}+M_{n-4}, \quad M_{0}=0, M_{1}=1, M_{2}=1, M_{3}=2
$$

and

$$
R_{n}=R_{n-1}+R_{n-2}+R_{n-3}+R_{n-4}, \quad R_{0}=4, R_{1}=1, R_{2}=3, R_{3}=7
$$


respectively. More detail on these sequences can be found, for example, in [11], [14], [15], [19], [21] and [22].

The sequences $\left\{M_{n}\right\}_{n \geq 0}$ and $\left\{R_{n}\right\}_{n \geq 0}$ can be extended to negative subscripts by defining

$$
M_{-n}=-M_{-(n-1)}-M_{-(n-2)}-M_{-(n-3)}+M_{-(n-4)}
$$

and

$$
R_{-n}=-R_{-(n-1)}-R_{-(n-2)}-R_{-(n-3)}+R_{-(n-4)}
$$

for $n=1,2,3, \ldots$ respectively. Therefore, recurrences (1.3) and (1.4) hold for all integer $n$.

The following Table 2 presents the first few values of the Tetranacci and Tetranacci-Lucas numbers with positive and negative subscripts:

Table 2. Tetranacci and Tetranacci-Lucas Numbers with non-negative and negative indices

\begin{tabular}{cccccccccccccccc}
\hline$n$ & 0 & 1 & 2 & 3 & 4 & 5 & 6 & 7 & 8 & 9 & 10 & 11 & 12 & 13 & $\ldots$ \\
\hline$M_{n}$ & 0 & 1 & 1 & 2 & 4 & 8 & 15 & 29 & 56 & 108 & 208 & 401 & 773 & 1490 & $\ldots$ \\
$M_{-n}$ & 0 & 0 & 0 & 1 & -1 & 0 & 0 & 2 & -3 & 1 & 0 & 4 & -8 & 5 & $\ldots$ \\
$R_{n}$ & 4 & 1 & 3 & 7 & 15 & 26 & 51 & 99 & 191 & 367 & 708 & 1365 & 2631 & 5071 & $\ldots$ \\
$R_{-n}$ & 4 & -1 & -1 & -1 & 7 & -6 & -1 & -1 & 15 & -19 & 4 & -1 & 31 & -53 & $\ldots$ \\
\hline
\end{tabular}

It is well known that for all integers $n$, usual Tetranacci and Tetranacci-Lucas numbers can be expressed using Binet's formulas

$$
M_{n}=\frac{\alpha^{n+2}}{(\alpha-\beta)(\alpha-\gamma)(\alpha-\delta)}+\frac{\beta^{n+2}}{(\beta-\alpha)(\beta-\gamma)(\beta-\delta)}+\frac{\gamma^{n+2}}{(\gamma-\alpha)(\gamma-\beta)(\gamma-\delta)}+\frac{\delta^{n+2}}{(\delta-\alpha)(\delta-\beta)(\delta-\gamma)}
$$

(see for example [24] or [11])

or

$$
M_{n}=\frac{\alpha-1}{5 \alpha-8} \alpha^{n-1}+\frac{\beta-1}{5 \beta-8} \beta^{n-1}+\frac{\gamma-1}{5 \gamma-8} \gamma^{n-1}++\frac{\delta-1}{5 \delta-8} \delta^{n-1}
$$

(see for example [7]) and

$$
R_{n}=\alpha^{n}+\beta^{n}+\gamma^{n}+\delta^{n}
$$

respectively, where $\alpha, \beta, \gamma$ and $\delta$ are the roots of the equation $x^{4}-x^{3}-x^{2}-x-1=0$. Moreover,

$$
\begin{aligned}
& \alpha=\frac{1}{4}+\frac{1}{2} \omega+\frac{1}{2} \sqrt{\frac{11}{4}-\omega^{2}+\frac{13}{4} \omega^{-1}}, \\
& \beta=\frac{1}{4}+\frac{1}{2} \omega-\frac{1}{2} \sqrt{\frac{11}{4}-\omega^{2}+\frac{13}{4} \omega^{-1}} \\
& \gamma=\frac{1}{4}-\frac{1}{2} \omega+\frac{1}{2} \sqrt{\frac{11}{4}-\omega^{2}-\frac{13}{4} \omega^{-1}} \\
& \delta=\frac{1}{4}-\frac{1}{2} \omega-\frac{1}{2} \sqrt{\frac{11}{4}-\omega^{2}-\frac{13}{4} \omega^{-1}},
\end{aligned}
$$


where

$$
\omega=\sqrt{\frac{11}{12}+\left(\frac{-65}{54}+\sqrt{\frac{563}{108}}\right)^{1 / 3}+\left(\frac{-65}{54}-\sqrt{\frac{563}{108}}\right)^{1 / 3}} .
$$

Note that the Binet form of a sequence satisfying (1.3) and (1.4) for non-negative integers is valid for all integers $n$. This result of Howard and Saidak [12] is even true in the case of higher-order recurrence relations.

The generating functions for the Tetranacci sequence $\left\{M_{n}\right\}_{n \geq 0}$ and Tetranacci-Lucas sequence $\left\{R_{n}\right\}_{n \geq 0}$ are

respectively.

$$
\sum_{n=0}^{\infty} M_{n} x^{n}=\frac{x}{1-x-x^{2}-x^{3}-x^{4}} \text { and } \sum_{n=0}^{\infty} R_{n} x^{n}=\frac{4-3 x-2 x^{2}-x^{3}}{1-x-x^{2}-x^{3}-x^{4}}
$$

\section{The Bicomplex Tetranacci and Tetranacci-Lucas Quaternions and their Generating}

\section{Functions, Binet's Formulas and Summations Formulas}

In this section we define the bicomplex Tetranacci and Tetranacci-Lucas quaternions and give generating functions and Binet formulas for them. First, we give some information about bicomplex type quaternion sequences from the literature.

Nurkan and Güven [16] (see also [17]) introduced $n$th bicomplex Fibonacci and $n$th bicomplex Lucas numbers (quaternions) as

$$
B F_{n}=F_{n}+F_{n+1} i+F_{n+2} j+F_{n+3} i j
$$

and

$$
B L_{n}=L_{n}+L_{n+1} i+L_{n+2} j+L_{n+3} i j
$$

respectively, where $F_{n}$ and $L_{n}$ are the $n$th Fibonacci and Lucas numbers respectively. Various families of bicomplex number (quaternion) sequences have been defined and studied by a number of authors. See, for example, $[1,2,4,9,10]$ for second order bicomplex quaternion sequences and $[5,13]$ for third order bicomplex quaternion sequences.

We now define bicomplex Tetranacci and Tetranacci-Lucas quaternions over the algebra $\mathbb{B} \mathbb{C}$.

Definition 1. The nth bicomplex Tetranacci quaternion is

$$
\mathbb{B} \mathbb{C} M_{n}=M_{n}+i M_{n+1}+j M_{n+2}+i j M_{n+3}
$$

and the nth Tetranacci-Lucas quaternion is

$$
\mathbb{B C} R_{n}=R_{n}+i R_{n+1}+j R_{n+2}+i j R_{n+3} .
$$

It can be easily shown that $\left\{\mathbb{B C} M_{n}\right\}_{n \geq 0}$ and $\left\{\mathbb{B C C} R_{n}\right\}_{n \geq 0}$ can also be defined by the recurrence relations:

$$
\mathbb{B C} M_{n}=\mathbb{B C} M_{n-1}+\mathbb{B C} M_{n-2}+\mathbb{B C} M_{n-3}+\mathbb{B C} M_{n-4}
$$

and

$$
\mathbb{B C} R_{n}=\mathbb{B C} R_{n-1}+\mathbb{B} \mathbb{C} R_{n-2}+\mathbb{B C} R_{n-3}+\mathbb{B C C} R_{n-4}
$$


with the intial conditions

$$
\mathbb{B} \mathbb{C} M_{0}=i+j+2 i j, \quad \mathbb{B} C M_{1}=1+i+2 j+4 i j, \quad \mathbb{B} \mathbb{C} M_{2}=1+2 i+4 j+8 i j, \mathbb{B C} M_{3}=2+4 i+8 j+15 i j
$$

and

$\mathbb{B C} R_{0}=4+i+3 j+7 i j, \quad \mathbb{B} C R_{1}=1+3 i+7+15 i j, \quad \mathbb{B C} R_{2}=3+7 i+15 j+26 i j, \quad \mathbb{B C} R_{3}=7+15 i+26 j+51 i j$.

The sequences $\left\{\mathbb{B C} M_{n}\right\}_{n \geq 0}$ and $\left\{\mathbb{B} \mathbb{C} R_{n}\right\}_{n \geq 0}$ can be extended to negative subscripts by defining

$$
\mathbb{B C} M_{-n}=-\mathbb{B C} M_{-(n-1)}-\mathbb{B} \mathbb{C} M_{-(n-2)}-\mathbb{B C} M_{-(n-3)}+\mathbb{B} \mathbb{C} M_{-(n-4)}
$$

and

$$
\mathbb{B C} R_{-n}=-\mathbb{B} \mathbb{C} R_{-(n-1)}-\mathbb{B} \mathbb{C} R_{-(n-2)}-\mathbb{B} \mathbb{C} R_{-(n-3)}+\mathbb{B} \mathbb{C} R_{-(n-4)}
$$

for $n=1,2,3, \ldots$ respectively. Therefore, recurrences (2.3) and (2.4) hold for all integer $n$.

The first few bicomplex Tetranacci and Tetranacci-Lucas quaternions with positive subscript and negative subscript are given in the following Table 1 and Table 2:

Table 1 bicomplex Tetranacci quaternions Table 2 bicomplex Tetranacci-Lucas quaternions

\begin{tabular}{ccccccc}
\hline$n$ & $\mathbb{B} \mathbb{C} M_{n}$ & $\mathbb{B} \mathbb{C} M_{-n}$ & & $n$ & $\mathbb{B} \mathbb{C} R_{n}$ & $\mathbb{B} \mathbb{C} R_{-n}$ \\
\cline { 1 - 2 } \cline { 5 - 7 } 0 & $i+j+2 i j$ & $i+j+2 i j$ & & 0 & $4+i+3 j+7 i j$ & $4+i+3 j+7 i j$ \\
1 & $1+i+2 j+4 i j$ & $j+i j$ & & 1 & $1+3 i+7+15 i j$ & $-1+4 i+j+3 i j$ \\
2 & $1+2 i+4 j+8 i j$ & $i j$ & & 2 & $3+7 i+15 j+26 i j$ & $-1-i+4 j+i j$ \\
3 & $2+4 i+8 j+15 i j$ & 1 & & 3 & $7+15 i+26 j+51 i j$ & $-1-i-j+4 i j$ \\
4 & $4+8 i+15 j+29 i j$ & $-1+i$ & & 4 & $15+26 i+51 j+99 i j$ & $7-i-j-i j$ \\
5 & $8+15 i+29 j+56 i j$ & $-i+j$ & & 5 & $26+51 i+99 j+191 i j$ & $-6+7 i-j-i j$ \\
6 & $15+29 i+56 j+108 i j$ & $-j+i j$ & & 6 & $51+99 i+191 j+367 i j$ & $-1-6 i+7 j-i j$ \\
7 & $29+56 i+108 j+208 i j$ & $2-i j$ & & 7 & $99+191 i+367 j+708 i j$ & $-1-i-6 j+7 i j$ \\
\hline
\end{tabular}

For two bicomplex Tetranacci quaternions $\mathbb{B C} M_{n}$ and $\mathbb{B} \mathbb{C} M_{k}$ and for skaler $\lambda \in \mathbb{R}$, the addition, substraction and multiplication with scalar are defined as componentwise, i.e.,

$$
\begin{aligned}
\mathbb{B} C M_{n}+\mathbb{B} \mathbb{C} M_{k} & =\left(M_{n}+M_{k}\right)+i\left(M_{n+1}+M_{k+1}\right)+j\left(M_{n+2}+M_{k+2}\right)+i j\left(M_{n+3}+M_{k+3}\right), \\
\mathbb{B} \mathbb{C} M_{n}-\mathbb{B C} M_{k} & =\left(M_{n}-M_{k}\right)+i\left(M_{n+1}-M_{k+1}\right)+j\left(M_{n+2}-M_{k+2}\right)+i j\left(M_{n+3}-M_{k+3}\right), \\
\lambda \mathbb{B} \mathbb{C} M_{n} & =\lambda M_{n}+i \lambda M_{n+1}+j \lambda M_{n+2}+i j \lambda M_{n+3}
\end{aligned}
$$


respectively, and product (multiplication) is defined as follows:

$$
\begin{aligned}
\mathbb{B C} M_{n} \times \mathbb{B} \mathbb{C} M_{k}= & \left(M_{n} M_{k}-M_{n+1} M_{k+1}-M_{n+2} M_{k+2}+M_{n+3} M_{k+3}\right) \\
& +i\left(M_{n} M_{k+1}+M_{n+1} M_{k}-M_{n+2} M_{k+3}-M_{n+3} M_{k+2}\right) \\
& +j\left(M_{n} M_{k+2}-M_{n+1} M_{k+3}+M_{n+2} M_{k}-M_{n+3} M_{k+1}\right) \\
& +i j\left(M_{n} M_{k+3}+M_{n+1} M_{k+2}+M_{n+2} M_{k+1}+M_{n+3} M_{k}\right) \\
= & \mathbb{B C} M_{k} \times \mathbb{B} \mathbb{C} M_{n} .
\end{aligned}
$$

Similarly, for two bicomplex Tetranacci-Lucas quaternions $\mathbb{B} \mathbb{C} R_{n}$ and $\mathbb{B} \mathbb{C} R_{k}$ and for skaler $\lambda \in \mathbb{R}$, the addition, substraction and multiplication with scalar are defined as componentwise, i.e.,

$$
\begin{aligned}
\mathbb{B} \mathbb{C} R_{n} \pm \mathbb{B} \mathbb{C} R_{k} & =\left(R_{n} \pm R_{k}\right)+i\left(R_{n+1} \pm R_{k+1}\right)+j\left(R_{n+2} \pm R_{k+2}\right)+i j\left(R_{n+3} \pm R_{k+3}\right) \\
\lambda \mathbb{B} \mathbb{C} R_{n} & =\lambda R_{n}+i \lambda R_{n+1}+j \lambda R_{n+2}+i j \lambda R_{n+3}
\end{aligned}
$$

respectively, and product (multiplication) is defined as follows:

$$
\begin{aligned}
\mathbb{B C C} R_{n} \times \mathbb{B} \mathbb{C} R_{k}= & \left(R_{n} R_{k}-R_{n+1} R_{k+1}-R_{n+2} R_{k+2}+R_{n+3} R_{k+3}\right) \\
& +i\left(R_{n} R_{k+1}+R_{n+1} R_{k}-R_{n+2} R_{k+3}-R_{n+3} R_{k+2}\right) \\
& +j\left(R_{n} R_{k+2}-R_{n+1} R_{k+3}+R_{n+2} R_{k}-R_{n+3} R_{k+1}\right) \\
& +i j\left(R_{n} R_{k+3}+R_{n+1} R_{k+2}+R_{n+2} R_{k+1}+R_{n+3} R_{k}\right) \\
= & \mathbb{B} \mathbb{C} R_{k} \times \mathbb{B} \mathbb{C} R_{n} .
\end{aligned}
$$

Note that

$$
\begin{aligned}
\mathbb{B C} M_{n} \times \mathbb{B} \mathbb{C} M_{n}= & \left(M_{n}^{2}-M_{n+1}^{2}-M_{n+2}^{2}+M_{n+3}^{2}\right)+2 i\left(M_{n} M_{n+1}-M_{n+2} M_{n+3}\right) \\
& +2 j\left(M_{n} M_{n+2}-M_{n+1} M_{n+3}\right)+2 i j\left(M_{n} M_{n+3}+M_{n+1} M_{n+2}\right)
\end{aligned}
$$

and

$$
\begin{aligned}
\mathbb{B} \mathbb{C} R_{n} \times \mathbb{B} \mathbb{C} R_{n}= & \left(R_{n}^{2}-R_{n+1}^{2}-R_{n+2}^{2}+R_{n+3}^{2}\right)+2 i\left(R_{n} R_{n+1}-R_{n+2} R_{n+3}\right) \\
& +2 j\left(R_{n} R_{n+2}-R_{n+1} R_{n+3}\right)+2 i j\left(R_{n} R_{n+3}+R_{n+1} R_{n+2}\right) .
\end{aligned}
$$

Moreover, three different conjugations for the bicomplex Tribonacci quaternion $\mathbb{B} \mathbb{C} M_{n}=M_{n}+i M_{n+1}+$ $j M_{n+2}+i j M_{n+3}$ are given as

$$
\begin{aligned}
\left(\mathbb{B C} M_{n}\right)_{i}^{*} & =M_{n}-i M_{n+1}+j M_{n+2}-i j M_{n+3}, \\
\left(\mathbb{B C} M_{n}\right)_{j}^{*} & =M_{n}+i M_{n+1}-j M_{n+2}-i j M_{n+3}, \\
\left(\mathbb{B C} M_{n}\right)_{i j}^{*} & =M_{n}-i M_{n+1}-j M_{n+2}+i j M_{n+3},
\end{aligned}
$$


and the squares of norms of the bicomplex Tribonacci quaternion are given by

$$
\begin{aligned}
N_{i}^{2}\left(\mathbb{B C} M_{n}\right) & =\left|\left(\mathbb{B C} M_{n}\right)_{i} \times\left(\mathbb{B C} M_{n}\right)_{i}^{*}\right|:=\left|M_{n}^{2}+M_{n+1}^{2}-M_{n+2}^{2}-M_{n+3}^{2}+2 j\left(M_{n} M_{n+2}+M_{n+1} M_{n+3}\right)\right|, \\
N_{j}^{2}\left(\mathbb{B} \mathbb{C} M_{n}\right) & =\left|\left(\mathbb{B C} M_{n}\right)_{j} \times\left(\mathbb{B C} M_{n}\right)_{j}^{*}\right|:=\left|M_{n}^{2}+M_{n+1}^{2}-M_{n+2}^{2}-M_{n+3}^{2}+2 i\left(M_{n} M_{n+1}+M_{n+2} M_{n+3}\right)\right|, \\
N_{i j}^{2}\left(\mathbb{B} \mathbb{C} M_{n}\right) & =\left|\left(\mathbb{B C} M_{n}\right)_{i j} \times\left(\mathbb{B C} M_{n}\right)_{i j}^{*}\right|:=\left|M_{n}^{2}+M_{n+1}^{2}+M_{n+2}^{2}+M_{n+3}^{2}+2 i j\left(M_{n} M_{n+3}-M_{n+2} M_{n+1}\right)\right| .
\end{aligned}
$$

Similarly, we can give three different conjugations and the squares of norms for the bicomplex TribonacciLucas quaternion $\mathbb{B} \mathbb{C} R_{n}=R_{n}+i R_{n+1}+j R_{n+2}+i j R_{n+3}$.

Now, we will state Binet's formula for the bicomplex Tetranacci and Tetranacci-Lucas quaternions and in the rest of the paper we fix the following notations.

$$
\begin{aligned}
& \widehat{\alpha}=1+i \alpha+j \alpha^{2}+i j \alpha^{3}, \\
& \widehat{\beta}=1+i \beta+j \beta^{2}+i j \beta^{3}, \\
& \widehat{\gamma}=1+i \gamma+j \gamma^{2}+i j \gamma^{3}, \\
& \widehat{\delta}=1+i \delta+j \delta^{2}+i j \delta^{3} .
\end{aligned}
$$

TheOREM 2. (Binet's Formulas) For any integer $n$, the nth bicomplex Tetranacci quaternion is

$$
\begin{aligned}
\mathbb{B C} M_{n}= & \frac{\widehat{\alpha} \alpha^{n+2}}{(\alpha-\beta)(\alpha-\gamma)(\alpha-\delta)}+\frac{\widehat{\beta} \beta^{n+2}}{(\beta-\alpha)(\beta-\gamma)(\beta-\delta)} \\
& +\frac{\widehat{\gamma} \gamma^{n+2}}{(\gamma-\alpha)(\gamma-\beta)(\gamma-\delta)}+\frac{\widehat{\delta} \delta^{n+2}}{(\delta-\alpha)(\delta-\beta)(\delta-\gamma)} \\
= & \frac{\alpha-1}{5 \alpha-8} \widehat{\alpha} \alpha^{n-1}+\frac{\beta-1}{5 \beta-8} \widehat{\beta} \beta^{n-1}+\frac{\gamma-1}{5 \gamma-8} \widehat{\gamma} \gamma^{n-1}+\frac{\delta-1}{5 \delta-8} \widehat{\delta} \delta^{n-1}
\end{aligned}
$$

and the nth bicomplex Tetranacci-Lucas quaternion is

$$
\mathbb{B} \mathbb{C} R_{n}=\widehat{\alpha} \alpha^{n}+\widehat{\beta} \beta^{n}+\widehat{\gamma} \gamma^{n}+\widehat{\delta} \delta^{n}
$$

Proof. Using Binet's formula of the Tetranacci-Lucas numbers we have

$$
\begin{aligned}
\mathbb{B C} R_{n}= & R_{n}+i R_{n+1}+j R_{n+2}+i j R_{n+3} \\
= & \left(\alpha^{n}+\beta^{n}+\gamma^{n}+\delta^{n}\right)+i\left(\alpha^{n+1}+\beta^{n+1}+\gamma^{n+1}+\delta^{n+1}\right) \\
& +j\left(\alpha^{n+2}+\beta^{n+2}+\gamma^{n+2}+\delta^{n+2}\right)+i j\left(\alpha^{n+3}+\beta^{n+3}+\gamma^{n+3}+\delta^{n+3}\right) \\
= & \widehat{\alpha} \alpha^{n}+\widehat{\beta} \beta^{n}+\widehat{\gamma} \gamma^{n}+\widehat{\delta} \delta^{n} .
\end{aligned}
$$


Note that using Binet's formula (1.5) of the Tetranacci numbers we have

$$
\begin{aligned}
\mathbb{B C} M_{n}= & M_{n}+i M_{n+1}+j M_{n+2}+i j M_{n+3} \\
= & \left(\frac{\alpha-1}{5 \alpha-8} \alpha^{n-1}+\frac{\beta-1}{5 \beta-8} \beta^{n-1}+\frac{\gamma-1}{5 \gamma-8} \gamma^{n-1}+\frac{\delta-1}{5 \delta-8} \delta^{n-1}\right) \\
& +i\left(\frac{\alpha-1}{5 \alpha-8} \alpha^{n}+\frac{\beta-1}{5 \beta-8} \beta^{n}+\frac{\gamma-1}{5 \gamma-8} \gamma^{n}+\frac{\delta-1}{5 \delta-8} \delta^{n}\right) \\
& +j\left(\frac{\alpha-1}{5 \alpha-8} \alpha^{n+1}+\frac{\beta-1}{5 \beta-8} \beta^{n+1}+\frac{\gamma-1}{5 \gamma-8} \gamma^{n+1}+\frac{\delta-1}{5 \delta-8} \delta^{n+1}\right) \\
& +i j\left(\frac{\alpha-1}{5 \alpha-8} \alpha^{n+2}+\frac{\beta-1}{5 \beta-8} \beta^{n+2}+\frac{\gamma-1}{5 \gamma-8} \gamma^{n+2}+\frac{\delta-1}{5 \delta-8} \delta^{n+2}\right) \\
= & \frac{\alpha-1}{5 \alpha-8} \widehat{\alpha} \alpha^{n-1}+\frac{\beta-1}{5 \beta-8} \widehat{\beta} \beta^{n-1}+\frac{\gamma-1}{5 \gamma-8} \widehat{\gamma} \gamma^{n-1}+\frac{\delta-1}{5 \delta-8} \widehat{\delta} \delta^{n-1} .
\end{aligned}
$$

This proves (2.6). Similarly, we can obtain (2.5).

Next, we present generating functions.

THEOREM 3. The generating functions for the bicomplex Tetranacci and Tetranacci-Lucas quaternions are

$$
\sum_{n=0}^{\infty} \mathbb{B} \mathbb{C} M_{n} x^{n}=\frac{(i+j+2 i j)+(1+j+2 i j) x+(j+2 i j) x^{2}+(j+i j) x^{3}}{1-x-x^{2}-x^{3}-x^{4}}
$$

and

$\sum_{n=0}^{\infty} \mathbb{B} \mathbb{C} R_{n} x^{n}=\frac{(4+i+3 j+7 i j)+(-3+2 i+4 j+8 i j) x+(-2+3 i+5 j+4 i j) x^{2}+(-1+4 i+j+3 i j) x^{3}}{1-x-x^{2}-x^{3}-x^{4}}$

respectively.

Proof. Let

$$
g(x)=\sum_{n=0}^{\infty} \mathbb{B} \mathbb{C} M_{n} x^{n}
$$

be generating function of the bicomplex Tetranacci quaternions. Then using the definition of the bicomplex Tetranacci quaternions, and substracting $x g(x), x^{2} g(x), x^{3} g(x)$ and $x^{4} g(x)$ from $g(x)$ and using the recurrence relation $\mathbb{B C} M_{n}=\mathbb{B C} M_{n-1}+\mathbb{B} \mathbb{C} M_{n-2}+\mathbb{B C} M_{n-3}+\mathbb{B} \mathbb{C} M_{n-4}$, we obtain

$$
\begin{aligned}
\left(1-x-x^{2}-x^{3}-x^{4}\right) g(x)= & \mathbb{B} \mathbb{C} M_{0}+\left(\mathbb{B C} M_{1}-\mathbb{B C} M_{0}\right) x+\left(\mathbb{B C} M_{2}-\mathbb{B C} M_{1}-\mathbb{B C} M_{0}\right) x^{2} \\
& +\left(\mathbb{B C} M_{3}-\mathbb{B C C} M_{2}-\mathbb{B} \mathbb{C} M_{1}-\mathbb{B C} M_{0}\right) x^{3}
\end{aligned}
$$


Now using

$$
\begin{aligned}
\mathbb{B} C M_{-1} & =j+i j \\
\mathbb{B C} M_{0} & =i+j+2 i j \\
\mathbb{B C} M_{1} & =1+i+2 j+4 i j \\
\mathbb{B C} M_{2} & =1+2 i+4 j+8 i j \\
\mathbb{B C} M_{3} & =2+4 i+8 j+15 i j
\end{aligned}
$$

we obtain

$$
g(x)=\frac{(i+j+2 i j)+(1+j+2 i j) x+(j+2 i j) x^{2}+(j+i j) x^{3}}{1-x-x^{2}-x^{3}-x^{4}}
$$

Similarly, we can obtain (2.9).

Next we present some summation formulas of Tetranacci numbers.

LEMMA 4. For $n \geq 1$ we have the following formulas:
(a): $\sum_{p=1}^{n} M_{p}=\frac{1}{3}\left(M_{n+2}+2 M_{n}+M_{n-1}-1\right)$
(b): $\sum_{p=1}^{n} M_{2 p+1}=\frac{1}{3}\left(2 M_{2 n+2}+M_{2 n}-M_{2 n-1}-2\right)$
(c): $\sum_{p=1}^{n} M_{2 p}=\frac{1}{3}\left(2 M_{2 n+1}+M_{2 n-1}-M_{2 n-2}-2\right)$.

The above Lemma is given in Soykan [20, Corollary 2.7]. It now follows that for every integer $n \geq 0$,

$$
\begin{aligned}
\sum_{p=0}^{n} M_{p} & =M_{0}+\sum_{p=1}^{n} M_{p}=\frac{1}{3}\left(M_{n+2}+2 M_{n}+M_{n-1}-1\right), \\
\sum_{p=0}^{n} M_{2 p+1} & =M_{1}+\sum_{p=1}^{n} M_{2 p+1}=\frac{1}{3}\left(2 M_{2 n+2}+M_{2 n}-M_{2 n-1}+1\right) \\
\sum_{p=0}^{n} M_{2 p} & =M_{0}+\sum_{p=1}^{n} M_{2 p}=\frac{1}{3}\left(2 M_{2 n+1}+M_{2 n-1}-M_{2 n-2}-2\right) .
\end{aligned}
$$

In the following Lemma we present some summation formulas of Tetranacci-Lucas numbers.

LEMMA 5. For $n \geq 1$ we have the following formulas:
(a): $\sum_{p=1}^{n} R_{p}=\frac{1}{3}\left(R_{n+2}+2 R_{n}+R_{n-1}-10\right)$
(b): $\sum_{p=1}^{n} R_{2 p+1}=\frac{1}{3}\left(2 R_{2 n+2}+R_{2 n}-R_{2 n-1}-11\right)$
(c): $\sum_{p=1}^{n} R_{2 p}=\frac{1}{3}\left(2 R_{2 n+1}+R_{2 n-1}-R_{2 n-2}-2\right)$. 
The above Lemma is given in Soykan [20, Corollary 2.8]. It now follows that for every integer $n \geq 0$,

$$
\begin{aligned}
\sum_{p=0}^{n} R_{p} & =R_{0}+\sum_{p=1}^{n} R_{p}=\frac{1}{3}\left(R_{n+2}+2 R_{n}+R_{n-1}+2\right) \\
\sum_{p=0}^{n} R_{2 p+1} & =R_{1}+\sum_{p=1}^{n} R_{2 p+1}=+\frac{1}{3}\left(2 R_{2 n+2}+R_{2 n}-R_{2 n-1}-8\right) \\
\sum_{p=0}^{n} R_{2 p} & =R_{0}+\sum_{p=1}^{n} R_{2 p}=\frac{1}{3}\left(2 R_{2 n+1}+R_{2 n-1}-R_{2 n-2}+10\right) .
\end{aligned}
$$

Next we present some summation formulas of bicomplex Tetranacci quaternions.

THEOREM 6. For $n \geq 0$ we have the following formulas:

(a):

$$
\sum_{p=0}^{n} \mathbb{B} \mathbb{C} M_{p}=\frac{1}{3}\left(\mathbb{B} \mathbb{C} M_{n+2}+2 \mathbb{B} \mathbb{C} M_{n}+\mathbb{B} \mathbb{C} M_{n-1}-(1+i+4 j+7 i j)\right)
$$

(b):

$$
\sum_{p=0}^{n} \mathbb{B} \mathbb{C} M_{2 p+1}=\frac{1}{3}\left(2 \mathbb{B} \mathbb{C} M_{2 n+2}+\mathbb{B} \mathbb{C} M_{2 n}-\mathbb{B C} M_{2 n-1}+(1-2 i-2 j-5 i j)\right)
$$

(c):

$$
\sum_{p=0}^{n} \mathbb{B} \mathbb{C} M_{2 p}=\frac{1}{3}\left(2 \mathbb{B} \mathbb{C} M_{2 n+1}+\mathbb{B} \mathbb{C} M_{2 n-1}-\mathbb{B} \mathbb{C} M_{2 n-2}-(2-i+2 j+2 i j)\right) .
$$

Proof.

(a): Using (2.1) and (2.10), we obtain

$$
\begin{aligned}
\sum_{p=0}^{n} \mathbb{B} \mathbb{C} M_{p}= & \sum_{p=0}^{n} M_{p}+i \sum_{p=0}^{n} M_{p+1}+j \sum_{p=0}^{n} M_{p+2}+i j \sum_{p=0}^{n} M_{p+3} \\
= & \left(M_{0}+\ldots+M_{n}\right)+i\left(M_{1}+\ldots+M_{n+1}\right) \\
& +j\left(M_{2}+\ldots+M_{n+2}\right)+i j\left(M_{3}+\ldots+M_{n+2}\right) .
\end{aligned}
$$

and so

$$
\begin{aligned}
3 \sum_{p=0}^{n} \mathbb{B} \mathbb{C} M_{p}= & \left(M_{n+2}+2 M_{n}+M_{n-1}-1\right) \\
& +i\left(M_{n+3}+2 M_{n+1}+M_{n}-1-3 M_{0}\right) \\
& +j\left(M_{n+4}+2 M_{n+2}+M_{n+1}-1-3\left(M_{0}+M_{1}\right)\right) \\
& +i j\left(M_{n+5}+2 M_{n+3}+M_{n+2}-1-3\left(M_{0}+M_{1}+M_{2}\right)\right) \\
= & \mathbb{B} \mathbb{C} M_{n+2}+2 \mathbb{B} \mathbb{C} M_{n}+\mathbb{B} \mathbb{C} M_{n-1}+c
\end{aligned}
$$


where

$$
\begin{aligned}
c & =-1+i\left(-1-3 M_{0}\right)+j\left(-1-3\left(M_{0}+M_{1}\right)\right)+i j\left(-1-3\left(M_{0}+M_{1}+M_{2}\right)\right) \\
& =-1-i-4 j-7 i j .
\end{aligned}
$$

Hence

$$
\sum_{p=0}^{n} \mathbb{B} \mathbb{C} M_{p}=\frac{1}{3}\left(\mathbb{B C} M_{n+2}+2 \mathbb{B} \mathbb{C} M_{n}+\mathbb{B} \mathbb{C} M_{n-1}-(1+i+4 j+7 i j)\right) .
$$

This proves (2.16).

(b): and (c) follows from the identities (2.11) and (2.12).

In the following Theorem, we give some summation formulas of bicomplex Tetranacci-Lucas quaternions.

THEOREM 7. For $n \geq 0$ we have the following formulas:

(a):

$$
\sum_{p=0}^{n} \mathbb{B} \mathbb{C} R_{p}=\frac{1}{3}\left(\mathbb{B C C} R_{n+2}+2 \mathbb{B} \mathbb{C} R_{n}+\mathbb{B} \mathbb{C} R_{n-1}+(2-10 i-13 j-22 i j)\right) .
$$

(b):

$$
\sum_{p=0}^{n} \mathbb{B} \mathbb{C} R_{2 p+1}=\frac{1}{3}\left(2 \mathbb{B} \mathbb{C} R_{2 n+2}+\mathbb{B} \mathbb{C} R_{2 n}-\mathbb{B} \mathbb{C} R_{2 n-1}-(8+2 i+11 j+11 i j)\right)
$$

(c):

$$
\sum_{p=0}^{n} \mathbb{B} \mathbb{C} R_{2 p}=\frac{1}{3}\left(2 \mathbb{B} \mathbb{C} R_{2 n+1}+\mathbb{B} \mathbb{C} R_{2 n-1}-\mathbb{B} \mathbb{C} R_{2 n-2}+(10-8 i-2 j-11 i j)\right) .
$$

Proof.

(a): Using (2.3) and (2.13), we obtain (2.17).

(b): and (c) follows from the identities (2.14) and (2.15).

\section{Matrices and Determinants related with Tetranacci and Tetranacci-Lucas Quaternions}

We define the square matrix $B$ of order 4 as:

$$
B=\left(\begin{array}{llll}
1 & 1 & 1 & 1 \\
1 & 0 & 0 & 0 \\
0 & 1 & 0 & 0 \\
0 & 0 & 1 & 0
\end{array}\right)
$$

such that $\operatorname{det} B=-1$. 
Induction proof may be used to establish

$$
B^{n}=\left(\begin{array}{cccc}
M_{n+1} & M_{n}+M_{n-1}+M_{n-2} & M_{n}+M_{n-1} & M_{n} \\
M_{n} & M_{n-1}+M_{n-2}+M_{n-3} & M_{n-1}+M_{n-2} & M_{n-1} \\
M_{n-1} & M_{n-2}+M_{n-3}+M_{n-4} & M_{n-2}+M_{n-3} & M_{n-2} \\
M_{n-2} & M_{n-3}+M_{n-4}+M_{n-5} & M_{n-3}+M_{n-4} & M_{n-3}
\end{array}\right)
$$

Matrix formulation of $M_{n}$ and $R_{n}$ can be given as

$$
\left(\begin{array}{c}
M_{n+3} \\
M_{n+2} \\
M_{n+1} \\
M_{n}
\end{array}\right)=\left(\begin{array}{llll}
1 & 1 & 1 & 1 \\
1 & 0 & 0 & 0 \\
0 & 1 & 0 & 0 \\
0 & 0 & 1 & 0
\end{array}\right)^{n}\left(\begin{array}{c}
M_{3} \\
M_{2} \\
M_{1} \\
M_{0}
\end{array}\right)
$$

and

$$
\left(\begin{array}{c}
R_{n+3} \\
R_{n+2} \\
R_{n+1} \\
R_{n}
\end{array}\right)=\left(\begin{array}{llll}
1 & 1 & 1 & 1 \\
1 & 0 & 0 & 0 \\
0 & 1 & 0 & 0 \\
0 & 0 & 1 & 0
\end{array}\right)^{n}\left(\begin{array}{c}
R_{3} \\
R_{2} \\
R_{1} \\
R_{0}
\end{array}\right)
$$

Induction proofs may be used to establish the matrix formulations $M_{n}$ and $R_{n}$.

Now we define the matrices $B_{M}$ and $B_{R}$ as

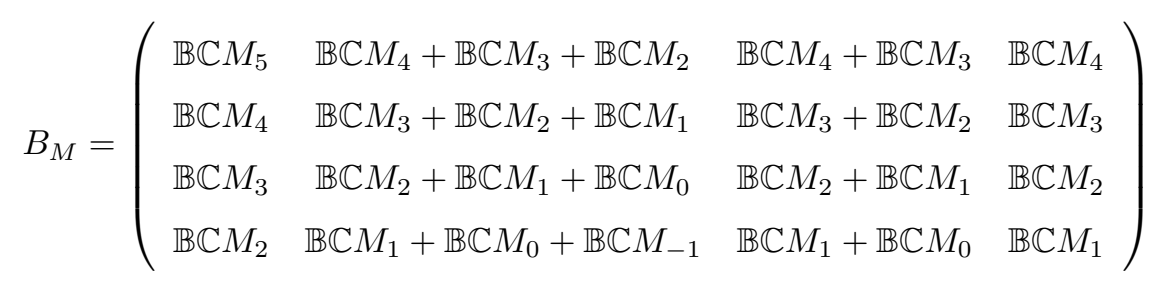

and

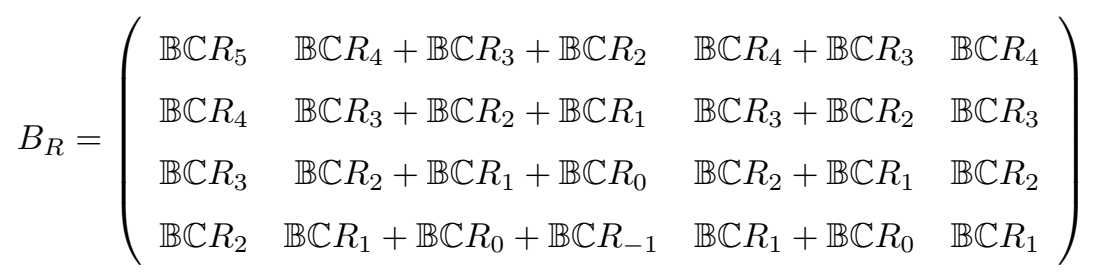

These matrices $B_{M}$ and $B_{R}$ can be called bicomplex Tetranacci quaternion matrix and bicomplex TetranacciLucas quaternion matrix, respectively.

THEOREM 8. For $n \geq 0$, the followings are valid: 
(a):

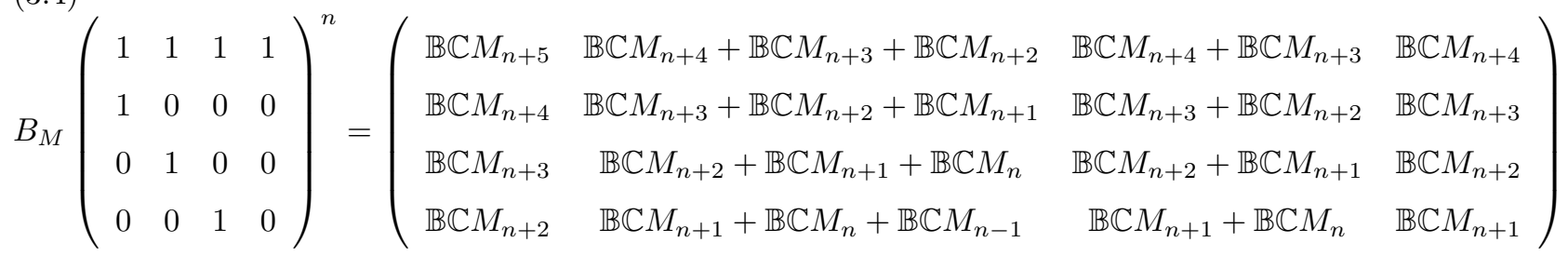

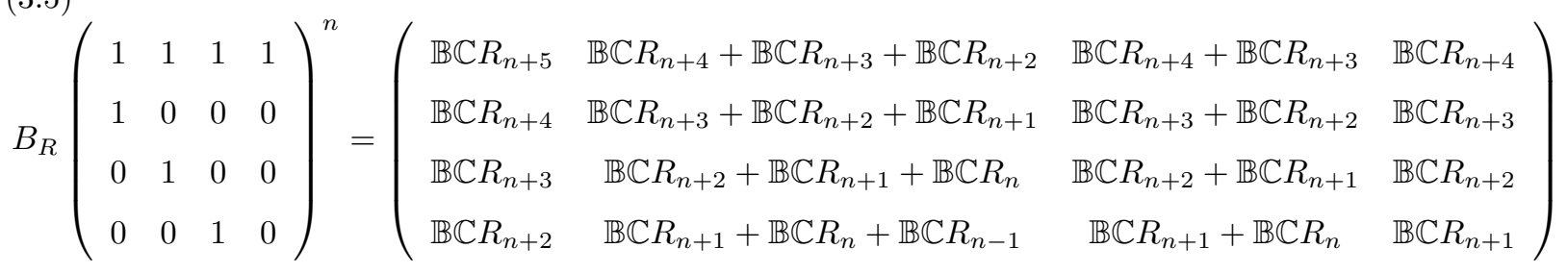

Proof. We prove (a) by mathematical induction on $n$. If $n=0$ then the result is clear. Now, we assume it is true for $n=k$, that is

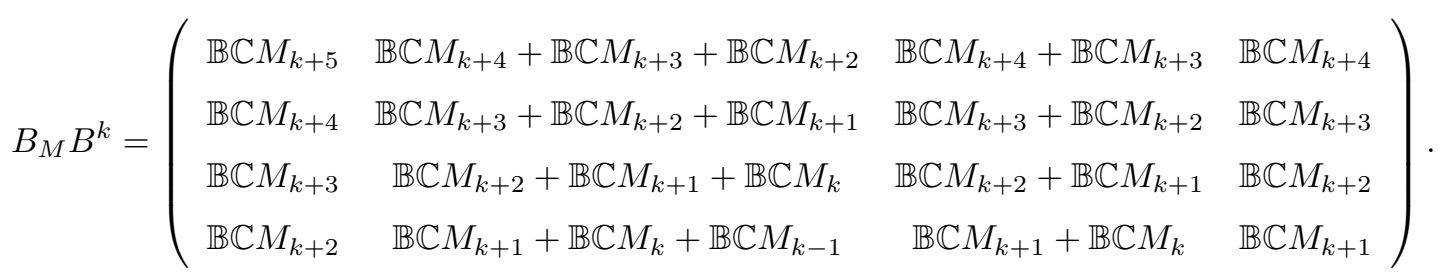

If we use (2.3), then we have $\mathbb{B C} M_{k+4}=\mathbb{B C} M_{k+3}+\mathbb{B C} M_{k+2}+\mathbb{B C} M_{k+1}+\mathbb{B C} M_{k}$. Then by induction hypothesis, we obtain

$$
\begin{aligned}
& B_{M} B^{k+1}=\left(B_{M} B^{k}\right) B
\end{aligned}
$$

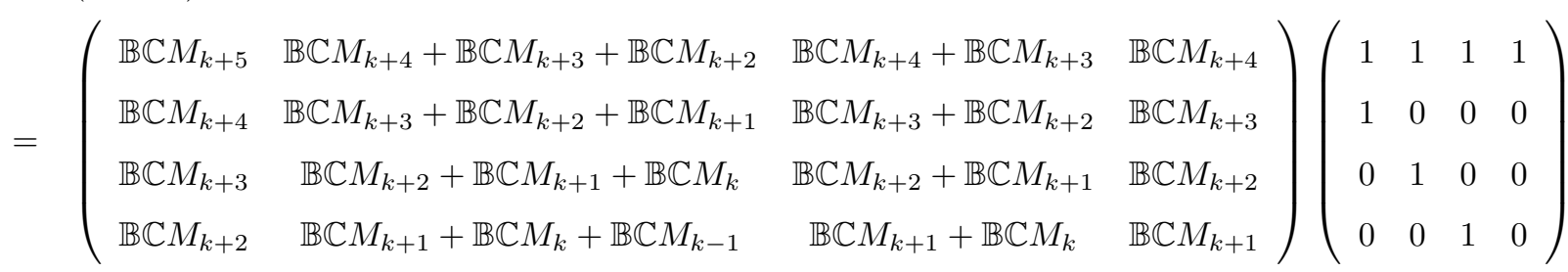

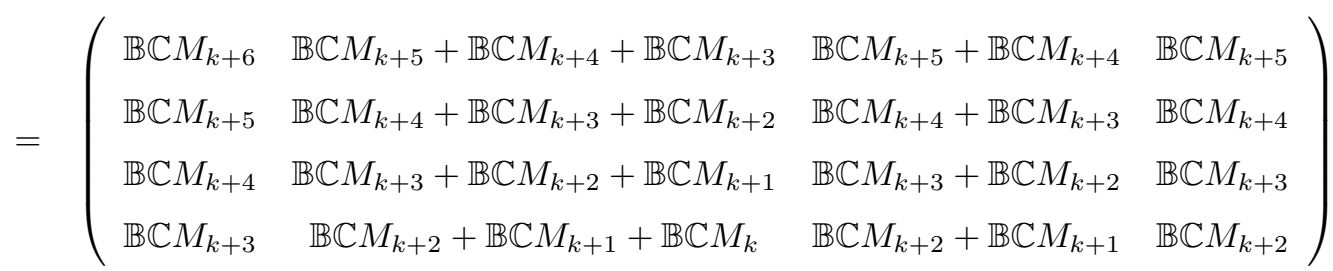

Thus, (3.4) holds for all non-negative integers $n$.

(3.5) can be similarly proved .

Corollary 9. For $n \geq 0$, the followings hold:

(a): $\mathbb{B C} M_{n+3}=\mathbb{B C} M_{3} M_{n+1}+\left(\mathbb{B C C} M_{2}+\mathbb{B C} M_{1}+\mathbb{B C} M_{0}\right) M_{n}+\left(\mathbb{B C} M_{1}+\mathbb{B C C} M_{2}\right) M_{n-1}+\mathbb{B} C M_{2} M_{n-2}$

(b): $\mathbb{B C C} R_{n+3}=\mathbb{B C} R_{3} M_{n+1}+\left(\mathbb{B C} R_{2}+\mathbb{B C} R_{1}+\mathbb{B C C} R_{0}\right) M_{n}+\left(\mathbb{B C} R_{1}+\mathbb{B C} R_{2}\right) M_{n-1}+\mathbb{B C} R_{2} M_{n-2}$. 
Proof. The proof of (a) can be seen by the coefficient of the matrix $B_{M}$ and (3.1). The proof of (b) can be seen by the coefficient of the matrix $B_{R}$ and (3.1).

\section{Five-Diagonal Matrix with Fourth Order Sequences and Applications}

In this section we give another way to obtain nth term of the bicomplex Tetranacci and Tetranacci-Lucas quaternions. For this we need the following theorem.

THEOREM 10. Let $\left\{x_{n}\right\}$ be any fourth-order linear sequence defined recursively as follows:

$$
x_{n}=r x_{n-1}+s x_{n-2}+t x_{n-3}+u x_{n-4}, \quad n \geq 4
$$

with the initial conditions $x_{0}=a, x_{1}=b, x_{2}=c, x_{3}=d$. Then for all $n \geq 0$, we have

$$
x_{n}=\left|\begin{array}{cccccccccccc}
a & -1 & 0 & 0 & 0 & 0 & 0 & \cdots & 0 & 0 & 0 & 0 \\
b & 0 & -1 & 0 & 0 & 0 & 0 & \cdots & 0 & 0 & 0 & 0 \\
c & 0 & 0 & -1 & 0 & 0 & 0 & \cdots & 0 & 0 & 0 & 0 \\
d & 0 & 0 & 0 & -1 & 0 & 0 & \cdots & 0 & 0 & 0 & 0 \\
0 & u & t & s & r & -1 & 0 & \cdots & 0 & 0 & 0 & 0 \\
0 & 0 & u & t & s & r & -1 & \cdots & 0 & 0 & 0 & 0 \\
\vdots & \ddots & \ddots & \ddots & \ddots & \ddots & \ddots & \ddots & \vdots & \vdots & \vdots & \vdots \\
0 & 0 & 0 & 0 & 0 & 0 & 0 & \ddots & s & r & -1 & 0 \\
0 & 0 & 0 & 0 & 0 & 0 & 0 & \ddots & t & s & r & -1 \\
0 & 0 & 0 & 0 & 0 & 0 & 0 & \ddots & u & t & s & r
\end{array}\right|_{(n+1) \times(n+1)}
$$

Proof. We proceed by induction on $n$. Since

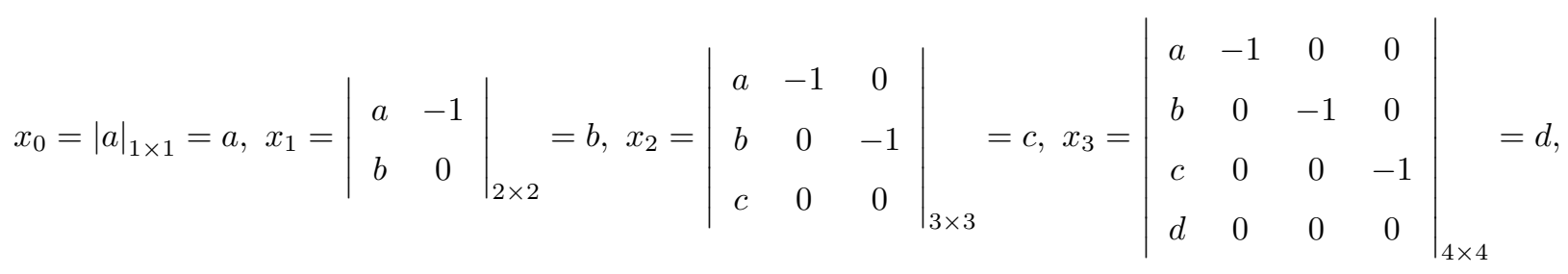


the equality holds for $n=0,1,2,3$. Now we assume that the equality is true for $4 \leq k \leq n$. Then we will complete the inductive step $n+1$ as follows: Note that

$$
x_{n+1}=\left|\begin{array}{ccccccccccc}
a & -1 & 0 & 0 & 0 & 0 & 0 & \cdots & 0 & 0 & 0 \\
b & 0 & -1 & 0 & 0 & 0 & 0 & \cdots & 0 & 0 & 0 \\
c & 0 & 0 & -1 & 0 & 0 & 0 & \cdots & 0 & 0 & 0 \\
d & 0 & 0 & 0 & -1 & 0 & 0 & \cdots & 0 & 0 & 0 \\
0 & u & t & s & r & -1 & 0 & \cdots & 0 & 0 & 0 \\
\vdots & \ddots & \ddots & \ddots & \ddots & \ddots & \ddots & \ddots & \vdots & \vdots & \vdots \\
0 & 0 & 0 & 0 & 0 & 0 & 0 & \ddots & r & -1 & 0 \\
0 & 0 & 0 & 0 & 0 & 0 & 0 & \ddots & s & r & -1 \\
0 & 0 & 0 & 0 & 0 & 0 & 0 & \ddots & t & s & r
\end{array}\right|_{(n+2) \times(n+2)}
$$

and

$$
\begin{aligned}
& x_{n+1}=r(-1)^{(n+2)+(n+2)}\left|\begin{array}{cccccccccc}
a & -1 & 0 & 0 & 0 & 0 & 0 & \cdots & 0 & 0 \\
b & 0 & -1 & 0 & 0 & 0 & 0 & \cdots & 0 & 0 \\
c & 0 & 0 & -1 & 0 & 0 & 0 & \cdots & 0 & 0 \\
d & 0 & 0 & 0 & -1 & 0 & 0 & \cdots & 0 & 0 \\
0 & u & t & s & r & -1 & 0 & \cdots & 0 & 0 \\
\vdots & \ddots & \ddots & \ddots & \ddots & \ddots & \ddots & \ddots & \vdots & \vdots \\
0 & 0 & 0 & 0 & 0 & 0 & 0 & \ddots & r & -1 \\
0 & 0 & 0 & 0 & 0 & 0 & 0 & \ddots & s & r
\end{array}\right|_{(n+1) \times(n+1)} \\
& +(-1)(-1)^{(n+2)+(n+1)}\left|\begin{array}{cccccccccc|c}
a & -1 & 0 & 0 & 0 & 0 & 0 & \cdots & 0 & 0 \\
b & 0 & -1 & 0 & 0 & 0 & 0 & \cdots & 0 & 0 \\
c & 0 & 0 & -1 & 0 & 0 & 0 & \cdots & 0 & 0 \\
d & 0 & 0 & 0 & -1 & 0 & 0 & \cdots & 0 & 0 \\
0 & u & t & s & r & -1 & 0 & \cdots & 0 & 0 \\
\vdots & \ddots & \ddots & \ddots & \ddots & \ddots & \ddots & \ddots & \vdots & \vdots \\
0 & 0 & 0 & 0 & 0 & 0 & 0 & \ddots & r & -1 \\
0 & 0 & 0 & 0 & 0 & 0 & 0 & \ddots & t & s
\end{array}\right|_{(n+1) \times(n+1)}
\end{aligned}
$$


and so

$$
\begin{aligned}
& x_{n+1}=r x_{n}+s\left|\begin{array}{ccccccccc}
a & -1 & 0 & 0 & 0 & 0 & 0 & \cdots & 0 \\
b & 0 & -1 & 0 & 0 & 0 & 0 & \cdots & 0 \\
c & 0 & 0 & -1 & 0 & 0 & 0 & \cdots & 0 \\
d & 0 & 0 & 0 & -1 & 0 & 0 & \cdots & 0 \\
0 & u & t & s & r & -1 & 0 & \cdots & 0 \\
\vdots & \ddots & \ddots & \ddots & \ddots & \ddots & \ddots & \ddots & \vdots \\
0 & 0 & 0 & 0 & 0 & 0 & 0 & \ddots & r
\end{array}\right|_{n \times n} \\
& +\left|\begin{array}{ccccccccc}
a & -1 & 0 & 0 & 0 & 0 & 0 & \cdots & 0 \\
b & 0 & -1 & 0 & 0 & 0 & 0 & \cdots & 0 \\
c & 0 & 0 & -1 & 0 & 0 & 0 & \cdots & 0 \\
d & 0 & 0 & 0 & -1 & 0 & 0 & \cdots & 0 \\
0 & u & t & s & r & -1 & 0 & \cdots & 0 \\
\vdots & \ddots & \ddots & \ddots & \ddots & \ddots & \ddots & \ddots & \vdots \\
0 & 0 & 0 & 0 & 0 & 0 & 0 & \ddots & t
\end{array}\right|_{n \times n}
\end{aligned}
$$




$$
\begin{aligned}
& x_{n}=r x_{n}+s x_{n-1}+t\left|\begin{array}{ccccccccc}
a & -1 & 0 & 0 & 0 & 0 & 0 & \cdots & 0 \\
b & 0 & -1 & 0 & 0 & 0 & 0 & \cdots & 0 \\
c & 0 & 0 & -1 & 0 & 0 & 0 & \cdots & 0 \\
d & 0 & 0 & 0 & -1 & 0 & 0 & \cdots & 0 \\
0 & u & t & s & r & -1 & 0 & \cdots & 0 \\
\vdots & \ddots & \ddots & \ddots & \ddots & \ddots & \ddots & \ddots & \vdots \\
0 & 0 & 0 & 0 & 0 & 0 & 0 & \ddots & t
\end{array}\right|_{(n-1) \times(n-1)} \\
& +\left|\begin{array}{ccccccccc}
a & -1 & 0 & 0 & 0 & 0 & 0 & \cdots & 0 \\
b & 0 & -1 & 0 & 0 & 0 & 0 & \cdots & 0 \\
c & 0 & 0 & -1 & 0 & 0 & 0 & \cdots & 0 \\
d & 0 & 0 & 0 & -1 & 0 & 0 & \cdots & 0 \\
0 & u & t & s & r & -1 & 0 & \cdots & 0 \\
\vdots & \ddots & \ddots & \ddots & \ddots & \ddots & \ddots & \ddots & \vdots \\
0 & 0 & 0 & 0 & 0 & 0 & 0 & \ddots & u
\end{array}\right|_{(n-1) \times(n-1)} \\
& =r x_{n}+s x_{n-1}+t x_{n-2}+\left|\begin{array}{ccccccccc}
a & -1 & 0 & 0 & 0 & 0 & 0 & \cdots & 0 \\
b & 0 & -1 & 0 & 0 & 0 & 0 & \cdots & 0 \\
c & 0 & 0 & -1 & 0 & 0 & 0 & \cdots & 0 \\
d & 0 & 0 & 0 & -1 & 0 & 0 & \cdots & 0 \\
0 & u & t & s & r & -1 & 0 & \cdots & 0 \\
\vdots & \ddots & \ddots & \ddots & \ddots & \ddots & \ddots & \ddots & \vdots \\
0 & 0 & 0 & 0 & 0 & 0 & 0 & \ddots & u
\end{array}\right|_{(n-1) \times(n-1)}
\end{aligned}
$$

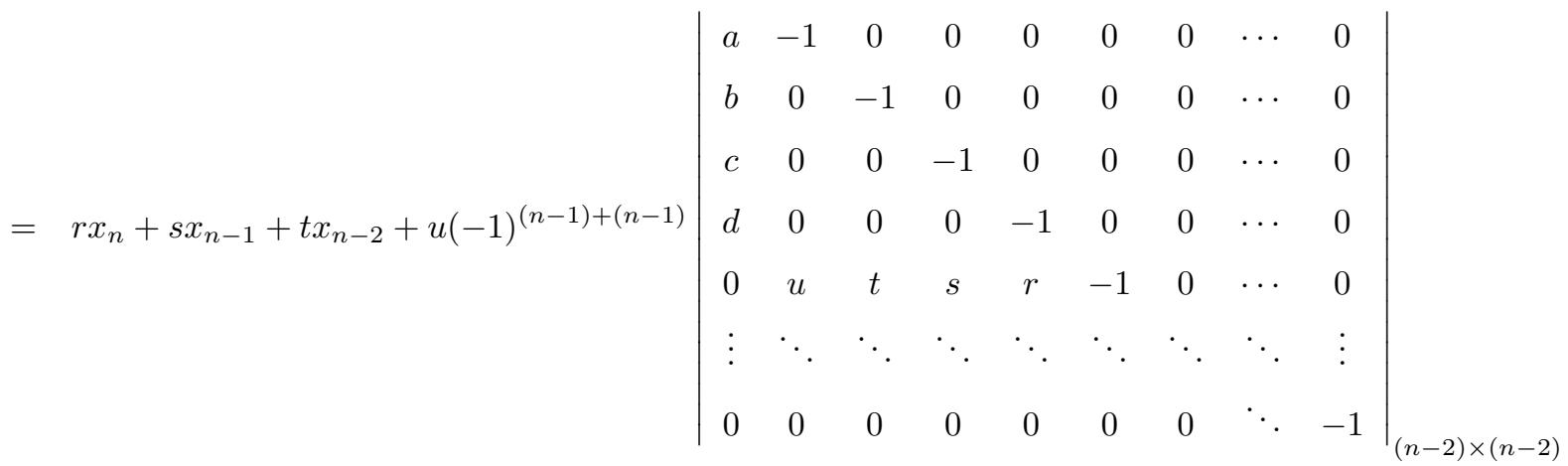

$$
\begin{aligned}
& =r x_{n}+s x_{n-1}+t x_{n-2}+u x_{n-3} \text {. }
\end{aligned}
$$

This completes the inductive step and the proof of the theorem.

Note that in our cases $r=s=t=u=1$. As a corallary of the above theorem, in the following we present another way to obtain nth term of the bicomplex Tetranacci and Tetranacci-Lucas quaternions. 
Corollary 11. For all $n \geq 0$, we have

(a):

$$
\mathbb{B} \mathbb{C} M_{n}=\left|\begin{array}{cccccccccccc|c}
\mathbb{B} \mathbb{C} M_{0} & -1 & 0 & 0 & 0 & 0 & 0 & \cdots & 0 & 0 & 0 & 0 & \\
\mathbb{B} \mathbb{C} M_{1} & 0 & -1 & 0 & 0 & 0 & 0 & \cdots & 0 & 0 & 0 & 0 & \\
\mathbb{B} \mathbb{C} M_{2} & 0 & 0 & -1 & 0 & 0 & 0 & \cdots & 0 & 0 & 0 & 0 & \\
\mathbb{B} \mathbb{C} M_{3} & 0 & 0 & 0 & -1 & 0 & 0 & \cdots & 0 & 0 & 0 & 0 & \\
0 & 1 & 1 & 1 & 1 & -1 & 0 & \cdots & 0 & 0 & 0 & 0 & \\
0 & 0 & 1 & 1 & 1 & 1 & -1 & \cdots & 0 & 0 & 0 & 0 \\
\vdots & \ddots & \ddots & \ddots & \ddots & \ddots & \ddots & \ddots & \vdots & \vdots & \vdots & \vdots \\
0 & 0 & 0 & 0 & 0 & 0 & 0 & \ddots & 1 & 1 & -1 & 0 & \\
0 & 0 & 0 & 0 & 0 & 0 & 0 & \ddots & 1 & 1 & 1 & -1 & \\
0 & 0 & 0 & 0 & 0 & 0 & 0 & \ddots & 1 & 1 & 1 & 1
\end{array}\right|_{(n+1) \times(n+1)}
$$

(b):

$$
\mathbb{B} \mathbb{C} R_{n}=\left|\begin{array}{cccccccccccc|c}
\mathbb{B} C R_{0} & -1 & 0 & 0 & 0 & 0 & 0 & \cdots & 0 & 0 & 0 & 0 \\
\mathbb{B} \mathbb{C} R_{1} & 0 & -1 & 0 & 0 & 0 & 0 & \cdots & 0 & 0 & 0 & 0 \\
\mathbb{B} \mathbb{C} R_{2} & 0 & 0 & -1 & 0 & 0 & 0 & \cdots & 0 & 0 & 0 & 0 & \\
\mathbb{B} \mathbb{C} R_{3} & 0 & 0 & 0 & -1 & 0 & 0 & \cdots & 0 & 0 & 0 & 0 \\
0 & 1 & 1 & 1 & 1 & -1 & 0 & \cdots & 0 & 0 & 0 & 0 \\
0 & 0 & 1 & 1 & 1 & 1 & -1 & \cdots & 0 & 0 & 0 & 0 \\
\vdots & \ddots & \ddots & \ddots & \ddots & \ddots & \ddots & \ddots & \vdots & \vdots & \vdots & \vdots \\
0 & 0 & 0 & 0 & 0 & 0 & 0 & \ddots & 1 & 1 & -1 & 0 \\
0 & 0 & 0 & 0 & 0 & 0 & 0 & \ddots & 1 & 1 & 1 & -1 \\
0 & 0 & 0 & 0 & 0 & 0 & 0 & \ddots & 1 & 1 & 1 & 1
\end{array}\right|_{(n+1) \times(n+1)}
$$

Proof. (a) follows from (2.3) and Theorem 10. (b) follows from (2.4) and Theorem 10.

\section{References}

[1] Aydın, F. T., Bicomplex k-Fibonacci Quaternions, arXiv: 1810.05003 [math.NT], 2018.

[2] Aydın, F. T., On Bicomplex Pell and Pell-Lucas Numbers, Communications in Advanced Mathematical Sciences, 1(2), 142-155, 2018.

[3] Baez, J., The octonions, Bull. Amer. Math. Soc. 39 (2), 145-205, 2002.

[4] Catarino, P., Bicomplex k-Pell Quaternions, Computational Methods and Function Theory, 2018. https://doi.org/10.1007/s40315-018-0251-5

[5] Cerda, G., Bicomplex Third-Order Jacobsthal quaternions, arXiv: 1809.06979 [math.AC], 2018.

[6] Conway, J. H., Smith, D.A.: On Quaternions and octonions: their geometry, arithmetic and symmetry. A K Peters/CRC Press, 2003. 
[7] Dresden, G. P., Du, Z., A Simplified Binet Formula for k-Generalized Fibonacci Numbers, J. Integer Seq. 17, art. 14.4.7, 9 pp., 2014.

[8] Luna-Elizarrarás, M.E., Shapiro, M., Struppa, D.C., Vajiac, A., Bicomplex Numbers and their Elementary Functions, CUBO A Mathematical Journal 14(2), 61-80, 2012.

[9] Halıcı, S., Karataş, A., Bicomplex Lucas and Horadam Numbers, arXiv: 1806.05038v2 [math.RA], 2018.

[10] Halici S. On Bicomplex Fibonacci Numbers and Their Generalization. In: Flaut C., Hošková-Mayerová Š., Flaut D. (eds) Models and Theories in Social Systems. Studies in Systems, Decision and Control, vol 179. Springer, pp 509-524, 2019.

[11] Hathiwala, G. S., Shah, D. V., Binet-Type Formula For The Sequence of Tetranacci Numbers by Alternate Methods, Mathematical Journal of Interdisciplinary Sciences 6 (1), 37-48, 2017.

[12] Howard, F.T., Saidak, F., Zhou's Theory of Constructing Identities, Congress Numer. 200, 225-237, 2010.

[13] Kızılateş, C., Catarino, P., Tuğlu, N., On the Bicomplex Generalized Tribonacci Quaternions. Mathematics 2019,7 , 80.

[14] Melham, R. S., Some Analogs of the Identity $F_{n}^{2}+F_{n+1}^{2}=F_{2 n+1}^{2}$, Fibonacci Quarterly, 305-311, 1999.

[15] Natividad, L. R., On Solving Fibonacci-Like Sequences of Fourth, Fifth and Sixth Order, International Journal of Mathematics and Computing, 3 (2), 2013.

[16] Nurkan, S. K., Güven İ, A., A Note on Bicomplex Fibonacci and Lucas Numbers, arXiv: 1508.03972 [math.NT], 2015.

[17] Nurkan, S. K., Güven İ, A., A Note on Bicomplex Fibonacci and Lucas Numbers, International Journal of Pure and Applied Mathematics, 120(3), 365-377, 2018.

[18] Rochon, D., Shapiro, M., On Algebraic Properties of Bicomplex and Hyperbolic Numbers. Anal. Univ. Oradea Fascicola. Matematica. 11, 1-28, 2004.

[19] Singh, B., Bhadouria, P., Sikhwal, O., Sisodiya, K., A Formula for Tetranacci-Like Sequence, Gen. Math. Notes, 20 (2), 136-141, 2014.

[20] Soykan, Y., Gaussian Generalized Tetranacci Numbers,. arXiv:1902.03936 [math.NT], 2019.

[21] Waddill, M. E., Another Generalized Fibonacci Sequence, Fibonacci Quarterly., 5 (3), 209-227, 1967.

[22] Waddill, M. E., The Tetranacci Sequence and Generalizations, The Fibonacci Quarterly, 9-20, 1992.

[23] Ward, J. P., Quaternions and Cayley Numbers: Algebra and Applications. Kluwer Academic Publishers, London, 1997.

[24] Zaveri, M. N., Patel, J. K., Binet's Formula for the Tetranacci Sequence, International Journal of Science and Research (IJSR), 5 (12), 1911-1914, 2016. 